\title{
Wald's martingale and the Moran process
}

Travis Monk ${ }^{1, *}$, André van $\operatorname{Schaik}^{1}$,

1 International Centre for Neuromorphic Systems, The MARCS Institute, Western Sydney University, Sydney, Australia

* travis.monk@westernsydney.edu.au

\section{Abstract}

Many models of evolution are stochastic processes, where some quantity of interest fluctuates randomly in time. One classic example is the Moran birth-death process, where that quantity is the number of mutants in a population. In such processes we are often interested in their absorption (i.e. fixation) probabilities, and the conditional distributions of absorption time. Those conditional time distributions can be very difficult to calculate, even for relatively simple processes like the Moran birth-death model. Instead of considering the time to absorption, we consider a closely-related quantity: the number of mutant population size changes before absorption. We use Wald's martingale to obtain the conditional characteristic functions of that quantity in the Moran process. Our expressions are novel, analytical, and exact. The parameter dependence of the characteristic functions is explicit, so it is easy to explore their properties in parameter space. We also use them to approximate the conditional characteristic functions of absorption time. We state the conditions under which that approximation is particularly accurate. Martingales are an elegant framework to solve principal problems of evolutionary stochastic processes. They do not require us to evaluate recursion relations, so we can quickly and tractably obtain absorption probabilities and times of evolutionary stochastic processes. 


\section{Author summary}

The Moran process is a probabilistic birth-death model of evolution. A mutant is introduced to an indigenous population, and we randomly choose organisms to live or die on subsequent time steps. Our goals are to calculate the probabilities that the mutant eventually dominates the population or goes extinct, and the distribution of time it requires to do so. The conditional distributions of time are difficult to obtain for the Moran process, so we consider a slightly different but related problem. We instead calculate the conditional distributions of the number of times that the mutant population size changes before it dominates the population or goes extinct. We use a martingale identified by Abraham Wald to obtain elegant and exact expressions for those distributions. We then use them to approximate conditional time distributions, and we show when that approximation is accurate. Our analysis outlines the basic concepts martingales and demonstrates why they are a formidable tool for studying probabilistic evolutionary models such as the Moran process.

\section{Introduction}

The Moran birth-death process is a classic model of evolution [1,2. It considers a population of two species that we call mutants and indigenous. On sequential time steps, one organism is chosen to reproduce from either species. The newborn is the same species as its parent, and it replaces another organism in the population uniformly at random. The total population size then remains constant, but the numbers of mutant and indigenous organisms in it are stochastic in time. The mutants will drive the indigenous organisms to extinction with some probability after some random number of time steps. Determining these probabilities and times are principal problems in the analysis of the Moran process 3, 4] and its extensions [5, 10].

The so-called 'fixation probability' of the Moran process is well-known 11 13]. However, the distribution of fixation time (and extinction time) is much harder to obtain. The means and variances of those distributions are known 5, 14, 15]. Their cumulants were recently reported, but only in the special cases of neutral selection or large population size 44. They are known to obey a particular symmetry [16]. We have 
closed-form expressions for those conditional time distributions, but they are written as $\quad 16$ sums of products of eigenvalues of submatrices [3]. The definition of the Moran process ${ }_{17}$ is simple, but calculating its conditional time distributions is difficult.

Martingales can provide the full distribution of absorption times for a specific class of stochastic processes 17, 18. Martingales are akin to conservation laws in physics. For example, in classical mechanics, we can invoke the conservation of energy to find the trajectory of a particle in a conserved system. Martingales are a statement that some quantity is conserved throughout a stochastic process. So if we know the value of that quantity at the beginning of the process, then we know it at the end. We can often calculate absorption (i.e. fixation) probabilities 8, 13 and/or times [17, 18 from it.

Abraham Wald identified a powerful martingale for stochastic processes whose steps are independent and identically distributed 17, 18. Wald's martingale is the seminal result of sequential analysis 19,20 . From that martingale, he obtained absorption probabilities and the conditional characteristic functions ( $\mathrm{CFs}$ ) of absorption times. We will show that Wald's martingale can be applied to the Moran process if we discard time steps where the mutant population size does not change. Therefore we can obtain the conditional CFs of the number of times that the mutant population size changes before it achieves fixation or goes extinct, i.e. 'active steps' 21. We will then use those CFs to approximate the CFs of the time to fixation and extinction, and we will show the conditions under which that approximation is particularly accurate. Our CFs for the number of mutant population size changes are novel, clean, and exact results. More generally, Wald's methodology demonstrates an elegant approach to investigate classic problems of evolutionary models $8,13,22$.

\section{Results}

Fig. 1 illustrates the problem considered by sequential analysis [19,20].

Let $S_{t}$ be the cumulative sum of $t$ realizations of independent and identically distributed (i.i.d.) random variables $X \sim \operatorname{Pr}(X)$ (Fig. 1 blue curves). Setting $S_{0}=0$, we write $S_{t}=\sum_{i=1}^{t} X_{i}$. On sequential time steps, we observe one realization of $X$ and add it to the sum of all previous realizations (Fig. 1. red dots). $S_{t}$ is then a random walk in time. 
Fig 1. Schematic of sequential analysis. We initialize a cumulative sum to be $S_{0}=0$. On subsequent time steps, we make one observation of $X \sim \operatorname{Pr}(X)$. The $X$ are i.i.d. on each time step (blue curves). For illustrative purposes, we horizontally shift $\operatorname{Pr}(X)$ given $S_{t-1}$ (red dots). In this example, the peak of the blue curve is horizontally aligned with the red dot below it. The sequential sum $S_{t}$ of observations of $X$ is a random walk in time $t$. While $S_{t}$ remains between two absorbing barriers $a$ and $b$, we continue adding observations of $X$ to $S_{t}$. We are interested in the probabilities that $S_{t}$ hits either barrier first, and the distribution of the number of observations $T$ it required to get there. In this example, $S_{T}=a$, and $T=56$.

Define two constant finite absorbing barriers $a>0$ and $b<0$ (vertical dashed lines, Fig. 11. While $b<S_{t}<a$, we continue making new observations of $X$, one per time step, and adding them to the sum. We stop this sequential process when $S_{t}$ meets or exceeds either absorbing barrier (which happens in finite time [17]). We want to find the probability of hitting either barrier before the other, $\operatorname{Pr}\left(S_{T}=a\right)$ and $\operatorname{Pr}\left(S_{T}=b\right)$, i.e. 'absorption probabilities.' We also want to find the (conditional) distributions of the number of observations required to hit them, $\operatorname{Pr}\left(T \mid S_{T}=a\right)$ and $\operatorname{Pr}\left(T \mid S_{T}=b\right)$.

Wald derived these quantities from a martingale [17,23,24]:

$$
\mathbb{E}\left[e^{S_{t} h} \phi_{X}(h)^{-t} \mid S_{t-1}\right]=e^{S_{t-1} h} \phi_{X}(h)^{-(t-1)},
$$

where $\phi_{X}(h)$ is the moment generating function (MGF) of $X$ and $h$ is its independent variable. For the Moran process, $\phi_{X}(h)$ exists because $X$ can only assume the finite values $+1,0$, or -1 .

The quantity $\mathbb{E}\left[e^{S_{t} h} \phi_{X}(h)^{-t}\right]$ is conserved throughout a stochastic process whose steps are i.i.d. We illustrate this conservation by taking the expectation of both sides of Wald's martingale:

$$
\mathbb{E}\left[e^{S_{t} h} \phi_{X}(h)^{-t}\right]=\mathbb{E}\left[e^{S_{t-1} h} \phi_{X}(h)^{-(t-1)}\right]
$$

so by induction:

$$
\mathbb{E}\left[e^{S_{t} h} \phi_{X}(h)^{-t}\right]=\mathbb{E}\left[e^{S_{0} h} \phi_{X}(h)^{0}\right]=1
$$

Doob's optional stopping theorem 24,25$]$ states that a randomly-stopped martingale is 
still a martingale. Letting $T$ be a random variable:

$$
\mathbb{E}\left[e^{S_{T} h} \phi_{X}(h)^{-T}\right]=1
$$

Eq. 1 is known as the fundamental identity in sequential analysis 17,20 . It is a particularly powerful martingale because it is valid for all values of $h$. This property allows us to extract absorption probabilities and times from it by choosing special values of $h$.

Under weak assumptions, $\phi_{X}(h)$ is convex, so it crosses 1 at two points $h=0$ and $h=h_{0} \neq 0$ (Lemma 2, 17]). We extract absorption probabilities by inserting $h=h_{0}$ into Eq. 1 .

$$
\mathbb{E}\left[e^{S_{T} h_{0}}\right]=1=\operatorname{Pr}\left(S_{T}=a\right) e^{a h_{0}}+\operatorname{Pr}\left(S_{T}=b\right) e^{b h_{0}}
$$

The process absorbs in finite time under weak conditions [17], so we can insert $\operatorname{Pr}\left(S_{T}=b\right)=1-\operatorname{Pr}\left(S_{T}=a\right)$ and rearrange:

Wald 17 also showed that we can obtain the conditional CFs of $T$ from his martingale. Splitting the expectation in Eq. 1.

$$
\mathbb{E}\left[e^{h a} \phi_{X}(h)^{-T} \operatorname{Pr}\left(S_{T}=a \mid T\right)\right]+\mathbb{E}\left[e^{h b} \phi_{X}(h)^{-T} \operatorname{Pr}\left(S_{T}=b \mid T\right)\right]=1 .
$$

Since $\phi_{X}(h)$ is convex (under weak assumptions [17]), its logarithm has two real roots in $h$, and its derivative is nonzero at those roots. Then for imaginary $\tau,-\log \phi_{X}(h)=\tau$ has two complex roots $h_{1}(\tau)$ and $h_{2}(\tau)$ in the neighborhood of $\tau=0$.

Inserting $\phi_{X}\left(h_{1}(\tau)\right)=e^{-\tau}$ and $\phi_{X}\left(h_{2}(\tau)\right)=e^{-\tau}$ in the expectations above, we obtain a system of two equations:

$$
\begin{aligned}
& \mathbb{E}\left[e^{a h_{1}(\tau)} e^{\tau T} \operatorname{Pr}\left(S_{T}=a \mid T\right)\right]+\mathbb{E}\left[e^{b h_{1}(\tau)} e^{\tau T} \operatorname{Pr}\left(S_{T}=b \mid T\right)\right]=1 \\
& \mathbb{E}\left[e^{a h_{2}(\tau)} e^{\tau T} \operatorname{Pr}\left(S_{T}=a \mid T\right)\right]+\mathbb{E}\left[e^{b h_{2}(\tau)} e^{\tau T} \operatorname{Pr}\left(S_{T}=b \mid T\right)\right]=1,
\end{aligned}
$$


which can be written as conditional CFs of $T, \psi_{T \mid b}(\tau)$ and $\psi_{T \mid a}(\tau)$ :

$$
\begin{aligned}
& \alpha e^{a h_{1}(\tau)} \psi_{T \mid a}(\tau)+(1-\alpha) e^{b h_{1}(\tau)} \psi_{T \mid b}(\tau)=1 \\
& \alpha e^{a h_{2}(\tau)} \psi_{T \mid a}(\tau)+(1-\alpha) e^{b h_{2}(\tau)} \psi_{T \mid b}(\tau)=1
\end{aligned}
$$

We have two equations, so we can solve for both conditional CFs.

Wald's analysis is exact when $S_{T}$ hits the absorbing barriers $a$ and $b$ exactly [17, as in the Moran process.

\section{Applying Wald's martingale to the Moran process}

For an introduction to the Moran process, see 12 .

Consider the Moran birth-death process with a fixed population size $N$, and mutants with a relative reproductive advantage of $r$ with respect to indigenous individuals $\left[11\right.$ 13]. Let $S_{t-1}$ be the mutant population size on time step $t-1$, and let $X_{t}$ be the change in the mutant population size on time step $t$. Then $S_{t}=S_{0}+\sum_{i-1}^{t} X_{t}$, where $S_{0}$ is the initial mutant population size.

Let $p_{X \uparrow}, p_{X \downarrow}$, and $p_{X 0}$ represent the Moran process transition probabilities, and let $F_{t-1}=r S_{t-1}+N-S_{t-1}$ represent the total fitness of the graph on time step $t-1$. The distribution of $X_{t}$ is:

$$
p_{X \uparrow}=\frac{r S_{t-1}}{F_{t-1}} \frac{N-S_{t-1}}{N} ; \quad p_{X \downarrow}=\frac{N-S_{t-1}}{F_{t-1}} \frac{S_{t-1}}{N} ; \quad p_{X 0}=1-p_{X \uparrow}-p_{X \downarrow} .
$$

We cannot directly apply Wald's martingale to the Moran process because the $X_{t}$ are not independent. The distribution of $X_{t}$ depends on the state $S_{t-1}$, and therefore on previous observations of $X$.

Instead, consider the random variable $Y_{t} \equiv X_{t} \mid\left(X_{t} \neq 0\right)$. In doing so we eliminate time steps where no change in the mutant population size occurs, i.e. we only consider 'active steps' 21. Redefine $S_{t}=S_{0}+\sum_{i=1}^{t} Y_{i}$, and let $p_{Y \uparrow}$ and $p_{Y \downarrow}$ represent transition probabilities:

$$
p_{Y \uparrow}=\frac{\operatorname{Pr}\left(X_{t}=1, X_{t} \neq 0\right)}{\operatorname{Pr}\left(X_{t} \neq 0\right)}=\frac{\operatorname{Pr}\left(X_{t}=1\right)}{1-\operatorname{Pr}\left(X_{t}=0\right)}=\frac{r}{r+1} ; \quad p_{Y \downarrow}=\frac{1}{r+1} .
$$

The transition probabilities of $Y_{t}$ are independent and identical [4, 6]. Therefore Wald's 80 
martingale is applicable to the Moran process if we eliminate time steps where the mutant population size does not change.

Eliminating time steps where $X_{t}=0$ does not affect the fixation probability of the Moran process. We can show this by evaluating Eq. 2. First we find the value $h=h_{0} \neq 0$ such that $\phi_{Y}\left(h_{0}\right)=1$ :

$$
\phi_{Y}\left(h_{0}\right)=\mathbb{E}\left[e^{h_{0} Y}\right]=\frac{r}{r+1} e^{h_{0}}+\frac{1}{r+1} e^{-h_{0}}=1 \quad \rightarrow \quad h_{0}=-\ln (r) .
$$

Typically we would set the Moran absorbing barriers to be $a=N$ (fixation) and $b=0$ (extinction), and we would set $S_{0}$ to be some value between them. However, Wald's analysis assumes that $S_{0}=0$, so we shift the barriers to $a=N-S_{0}$ and $b=-S_{0}$. Inserting $h_{0}, a$, and $b$ into Eq. 2 .

$$
\alpha=\frac{1-e^{S_{0} \ln (r)}}{e^{\left(S_{0}-N\right) \ln (r)}-e^{S_{0} \ln (r)}}=\frac{r^{-S_{0}}-1}{r^{-N}-1} .
$$

We recover the fixation probability of the Moran process $2,11-13$.

We can generalize Eq. 4 to find the probability that the Moran process achieves any state $a$ before $b$ starting from some $S_{0}$ between them. Call that probability $\alpha_{\left[b, S_{0}, a\right]}$ :

$$
\alpha_{\left[b, S_{0}, a\right]}=\frac{r^{-S_{0}}-r^{-b}}{r^{-a}-r^{-b}}
$$

We next use Eq. 3 to find the conditional CFs of the number of mutant population size changes to achieve absorption $C_{T}$. We begin by solving for the two roots $h_{1}(\tau)$ and $h_{2}(\tau)$ of $\phi_{Y}(h)=e^{-\tau}$ :

$$
\phi_{Y}(h)=\mathbb{E}\left[e^{Y h}\right]=\frac{r}{r+1} e^{h}+\frac{r}{r+1} e^{-h}=e^{-\tau},
$$

which has the analytical solution:

$$
h(\tau)=\ln \frac{(r+1) e^{-\tau} \pm \sqrt{(r+1)^{2} e^{-2 \tau}-4 r}}{2 r} .
$$

Fig. 2 plots these roots for the Moran process (Eq. 6) when $r=.7$ (top row) or $r=1.3$ (bottom row). The left column plots the MGF of $Y$, which crosses 1 at $h=0$ 
and $h_{0}=-\ln r$ (gray circles). The log of the MGF then has two complex roots $h_{1}(\tau)$

(middle column) and $h_{2}(\tau)$ (right column), where $\tau$ is purely imaginary. The real part

(red) and imaginary part (black) of the roots are plotted separately. When $\tau=0$, the

roots correspond to the two points where $\phi_{Y}(h)=1$. Specifically, $h_{1}(0)=0+0 i$, and

$h_{2}(0)=h_{0}+0 i$. These points are marked by the gray (imaginary part) and pink (real

part) circles, respectively.

Fig 2. The roots $h_{1}(\tau)$ and $h_{2}(\tau)$ (Eq. 6) in the complex plane. Left column: $\phi_{Y}(h)$ crosses 1 at two points for all $r \neq 1: h=0$ and $h=-\ln (r)$ (gray circles).

Therefore its logarithm has two roots in the complex plane if $r \neq 1$. Middle and right columns: real (red) and imaginary (black) parts of those roots $h_{1}(\tau)$ and $h_{2}(\tau)$,

respectively. When $\tau=0$, the imaginary parts of all roots are 0 (gray circles). The real parts of $h(0)$ are 0 (pink circles, middle column) or $-\ln (r)$ (pink circles, right column). Top row: $r=0.7$. Bottom row: $r=1.3$. Notice that the signs of the real and imaginary parts of both roots switch on either side of $r=1$.

Fig. 2 shows that the signs of the real and imaginary parts of the roots depend on whether $r<1$ or $r>1$. We must take care in identifying which root is which. By convention, $h_{1}$ is the root that passes through the origin of the complex plane. So if $r<1$, then $h_{1}(\tau)$ corresponds to subtraction and $h_{2}(\tau)$ corresponds to addition of the \pm sign in Eq. 6. and vice versa.

Fig. 3 shows the conditional CFs $\psi_{C_{T} \mid b}(\tau)$ (left plots) and $\psi_{C_{T} \mid a}(\tau)$ (right plots) that we obtain by inserting $h_{1}(\tau)$ and $h_{2}(\tau)$ into Eq. 3. We evaluated Eq. 3 for $r=0.9$ (top plots) and $r=1.5$ (bottom plots), and we used $N=10$ and $S_{0}=3$ in all plots. The real (pink) and imaginary (gray) parts of Eq. 3 are shown by the thick solid traces in Fig. 3 The real and imaginary parts of the CFs are even and odd, respectively, and they pass through 1 and 0 at $\tau=0$.

Fig 3. Conditional CFs for the number of changes in the mutant population size before absorption in the Moran process. CFs are conditional on the mutant population going extinct (left panels) or fixing (right panels). Real (pink) and imaginary (gray) parts are plotted separately. Fourier transforms of simulation results (red and black dashed lines) match theoretical predictions (thick pink and gray lines). The conditional first and second moments of $C_{T}$ are visualized (black line and red parabola) and their values are reported (black and red numbers). Parameters were $S_{0}=3, N=10$, and $r=.9$ (top row) or $r=1.5$ (bottom row).

Fig. 3 compares Eq. 3 with simulation results (dashed lines). We simulated the Moran process 100,000 times with the stated parameter values. We stored whether the initial mutant population fixed or went extinct, and how many times the mutant 
population size changed before doing so. We applied the Fourier transform to our simulated results to compare them with our predicted CFs. Their match is excellent because our analysis is exact, and we performed sufficiently many simulations for the Moran process to converge closely to that solution.

Notice that the conditional CFs in the top row of Fig. 3 are similar to those in the bottom row. Therefore the CFs of $C_{T}$ are not particularly sensitive to changes in $r$. Comparing the left column with the right column, we see that the CFs of $C_{T}$ are strongly influenced by where the Moran process absorbs. In particular, $\mathbb{E}\left[C_{T} \mid S_{T}=b\right]$ and $\mathbb{E}\left[C_{T} \mid S_{T}=a\right]$ are given by the slopes of the imaginary parts of the CFs at $\tau=0$ (thin solid lines, Fig. 3). The black numbers in Fig. 3 report these slopes. For these parameter values, fixation requires almost double the number of mutant population size changes as extinction does on average. We also include a visualization of the second moment (red parabolas) and report its value (red numbers). The second moment increases as the mean increases, but notice that the second moment reduces when $r$ is increased from 0.9 to 1.5 (c.f. red numbers, top and bottom row).

Fig. 4 plots conditional CFs $\psi_{C_{T} \mid b}(\tau)$ and $\psi_{C_{T} \mid a}(\tau)$ analogously to Fig. 3 , except we vary $N$ and $S_{0}$ as shown instead of $r(r=1.3)$. We again verify that those conditional CFs (thick pink and gray lines) match Fourier transforms of 100,000 simulations as described above (thin dashed lines).

Fig 4. Conditional CF dependence on $N$ and $S_{0}$. Conditional CFs and simulation results plotted as in Fig. 3. $N=10$ (left four plots) or 30 (right four plots), and $S_{0}=1$ (top row) or 3 (bottom row). $\psi_{C_{T} \mid a}(\tau)$ is sensitive to changes in $N$ but not $S_{0}$ if $S_{0} \ll N$ (the scale of the x-axis in the rightmost column is five times smaller than the others). $\psi_{C_{T} \mid b}(\tau)$ is sensitive to changes in $S_{0}$ but not $N . r=1.3$ in all plots. Simulations were run 100,000 times in each region of parameter space. The conditional first and second moments of $C_{T}$ are visualized and their numbers are reported (thin solid black and red lines and numbers).

Fig. 4 shows that $\psi_{C_{T} \mid b}(\tau)$ is not particularly sensitive to changes in $N$, but $\psi_{C_{T} \mid a}(\tau)$ is (notice that the x-axis of the right column has a different scale than the other columns). We see that increasing $N$ from 10 (left four plots) to 30 (right four plots) significantly increases the first two moments of $C_{T} \mid S_{T}=a$ (c.f. black and red numbers, second and fourth columns). This result reflects the fact that the Moran process requires more changes in the mutant population size to fix as the fixation barrier moves further from $S_{0}$. Increasing $N$ also increases $\mathbb{E}\left[C_{T} \mid S_{T}=b\right]$ because 
longer paths to extinction become possible. For example, if $N=10, S_{t}$ cannot increase from $S_{0}$ to 10 and then go extinct, because the mutant population already fixed. But if $N=30$, then that path to extinction becomes possible (albeit unlikely if $r>1$ ), and that path requires a large number of changes in the mutant population size.

Fig. 4 also shows that $\psi_{C_{T} \mid a}(\tau)$ is not particularly sensitive to changes in $S_{0}$, but $\psi_{C_{T} \mid b}(\tau)$ is. Increasing $S_{0}$ from 1 (top row) to 3 (bottom row) significantly increases the first two moments of $C_{T} \mid S_{T}=b$ (c.f. black and red numbers, first and third column). Again, the Moran process requires more changes in the mutant population size to go extinct as the extinction barrier moves further from $S_{0}$. Conversely, increasing $S_{0}$ slightly reduces the first two moments of $C_{T} \mid a$. However, slightly longer paths to fixation become possible if we increase $S_{0}$ as well. For example, if $S_{0}=3$, the mutant population can decrease to $S_{t}=1$ and then achieve fixation. That path is slightly longer on average than a path that starts at $S_{0}=1$ and achieves fixation. These two effects reduce each other's impact on $\psi_{C_{T} \mid a}(\tau)$ when $S_{0}$ is small.

\section{Approximating the conditional CFs of absorption time}

Fig. 5 shows that the conditional CFs of $C_{T}$ can be used to accurately approximate the conditional CFs of $T$. Fig. 5 plots the conditional CFs of $C_{T}$ with $r=1.3, N=10$, and $S_{0}=3$ (the lower-left panel of Fig. 4 and its neighbor to the right). We also plot the Fourier transform of 100,000 simulations where we stored the conditional time $T$ required to fix or go extinct (thin dashed lines). Real (pink and red) and imaginary (gray and black) parts are again plotted separately. We see that the conditional CFs of $C_{T}$ and $T$ are similar in form. The principal difference between them is the scale of $\tau$. In Fig. 5 the bottom x-axes correspond to $\psi_{C_{T} \mid b}(\tau)$ and $\psi_{C_{T} \mid a}(\tau)$, and the top x-axes correspond to $\psi_{T \mid b}(\tau)$ and $\psi_{T \mid a}(\tau)$.

Fig 5. The conditional CFs of $C_{T}$ can accurately approximate the conditional CFs of $T$. The main difference between them is the scale of $\tau$. The conditional CFs of $C_{T}$ are replotted from the two panels of Fig. 4 with $N=10$ and $S_{0}=3$. The thin dashed lines are Fourier transforms of 100,000 simulations of $T \mid b$ (left panel) and $T \mid a$ (right panel) using the same parameter values. Real (pink and red) and imaginary (gray and black) parts are plotted separately. Simulation results of $T$ are plotted on a different scale of $\tau$ (top x-axis markings) than the theoretical results of $C_{T}$ (bottom $\mathrm{x}$-axis markings). If we can find those scaling factors $\kappa_{a}$ (fixation) and $\kappa_{b}$ (extinction), then we can approximate $\psi_{T \mid a}(\tau)$ and $\psi_{T \mid b}(\tau)$ from $\psi_{C_{T} \mid a}(\tau)$ and $\psi_{C_{T} \mid b}(\tau)$. 
This observation indicates that the conditional CFs of $T$ can be approximated as:

$$
\psi_{T \mid b}(\tau) \approx \psi_{C_{T} \mid b}\left(\kappa_{b} \tau\right)=\psi_{\kappa_{b} C_{t} \mid b}(\tau) ; \quad \psi_{T \mid a}(\tau) \approx \psi_{C_{T} \mid a}\left(\kappa_{a} \tau\right)=\psi_{\kappa_{a} C_{T} \mid a}(\tau)
$$

where $\kappa_{b}$ and $\kappa_{a}$ are scaling constants conditional on extinction or fixation, respectively. $\quad{ }_{163}$

A natural choice for $\kappa_{b}$ and $\kappa_{a}$ is the expected waiting time per change $T_{C}$ in the mutant population size, conditional on fixation or extinction. Then we approximate $T$ as (number of mutant population size changes) · (expected time per change):

$$
T\left|a \approx C_{T}\right| a \cdot \mathbb{E}\left[T_{C} \mid a\right] ; \quad T\left|b \approx C_{T}\right| b \cdot \mathbb{E}\left[T_{C} \mid b\right] .
$$

We now calculate $\kappa_{a}=\mathbb{E}\left[T_{C} \mid a\right]$ (the calculation of $\kappa_{b}$ is analogous).

$\mathbb{E}\left[T_{C} \mid a\right]$ is the conditional expected time to absorption $\mathbb{E}[T \mid a]$ divided by the conditional expected number of mutant population changes $\mathbb{E}\left[C_{T} \mid a\right]$.

The conditional expected time to fixation is:

$$
\mathbb{E}[T \mid a]=\sum_{S_{t}=1}^{N-1} \mathbb{E}\left[V_{S} \mid a\right] \mathbb{E}\left[T_{V} \mid a\right],
$$

where $V_{S}$ is the number of visits to state $S_{t}$, and $T_{V}$ is the time spent per visit 6 . $\mathbb{E}\left[T_{C} \mid a\right]$ is then:

$$
\mathbb{E}\left[T_{C} \mid a\right]=\sum_{S_{t}=1}^{N-1} \frac{\mathbb{E}\left[V_{S} \mid a\right] \mathbb{E}\left[T_{V} \mid a\right]}{\mathbb{E}\left[C_{T} \mid a\right]} .
$$

We now calculate the three conditional expectations in the summand.

The conditional expected number of visits to transient states. We calculate $\mathbb{E}\left[V_{S} \mid a\right]$ by noting that $V_{S} \mid a$ is geometrically distributed 26 . Let $p_{V} \mid a$ be the probability that the mutant population fixes without returning to the state $S_{t}$ that it currently occupies. Let $R_{S_{t}}=0$ denote that occurrence. $p_{V} \mid a$ is then:

$$
p_{V} \mid a=\operatorname{Pr}\left(R_{S_{t}}=0 \mid S_{T}=a\right)=\frac{\operatorname{Pr}\left(S_{T}=a, R_{S_{t}}=0\right)}{\operatorname{Pr}\left(S_{T}=a\right)} .
$$

The only way that the Moran process can fix and not return to $S_{t}$ is that a) the process increases by 1 , and b) it then fixes without returning. We find these two absorption 
probabilities by inserting appropriate starting conditions and absorbing barriers into Eq.

5. For a) insert $S_{0}=S_{t}, a=S_{t}+1$, and $b=S_{t}-1$, and for b) insert $S_{0}=S_{t}+1$, $a=N$, and $b=S_{t}$ :

$$
\operatorname{Pr}\left(S_{T}=a, R_{S_{t}}=0\right)=\frac{r}{r+1} \cdot \frac{r^{-S_{t}-1}-r^{-S_{t}}}{r^{-N}-r^{-S_{t}}}
$$

$\operatorname{Pr}\left(S_{T}=a\right)$ is the fixation probability (Eq. 4), but starting from $S_{0}=S_{t} \cdot p_{V} \mid a$ is then:

$$
p_{V} \mid a=\frac{r}{r+1} \frac{r^{-S_{t}-1}-r^{-S_{t}}}{r^{-N}-r^{-S_{t}}} \frac{r^{-N}-1}{r^{-S_{t}}-1}
$$

$\mathbb{E}\left[V_{S} \mid a\right]=\left(p_{V} \mid a\right)^{-1}$, if a state was visited at least once before fixation. Since the Moran process fixes, we know that all states $S_{t} \geq S_{0}$ will be visited at least once. But for $S_{t}<S_{0}$, we need the probability that the process arrives at state $S_{t}$ at some time before it eventually fixes. Let $p_{A} \mid a$ represent that probability, and let $A_{S_{t}}=1$ represent that occurrence:

$$
p_{A} \mid a=\operatorname{Pr}\left(A_{S_{t}}=1 \mid S_{T}=a\right)=\frac{\operatorname{Pr}\left(A_{S_{t}}=1, S_{T}=a\right)}{\operatorname{Pr}\left(S_{T}=a\right)} .
$$

$\operatorname{Pr}\left(A_{S_{t}}=1, S_{T}=a\right)$ is the probability that the process, starting at $S_{0}$, a) absorbs at $S_{t}$ before $N$, and then b) absorbs at $N$ before 0 . For a) insert $S_{0}=S_{0}, a=S_{t}$, and $b=N$, and for b) insert $S_{0}=S_{t}, a=N$, and $b=0$ into Eq. $5 . \operatorname{Pr}\left(S_{T}=a\right)$ is given by Eq. 4. 4 . $p_{A} \mid a$ is then:

$$
p_{A} \mid a=\frac{r^{-S_{0}}-r^{-N}}{r^{-S_{t}}-r^{-N}} \frac{r^{-S_{t}}-1}{r^{-N}-1} \frac{r^{-N}-1}{r^{-S_{0}}-1}=\frac{r^{-S_{0}}-r^{-N}}{r^{-S_{t}}-r^{-N}} \frac{r^{-S_{t}}-1}{r^{-S_{0}-1}} .
$$

We can now find $\mathbb{E}\left[V_{S} \mid a\right]$ :

$$
\mathbb{E}\left[V_{S} \mid a\right]=1 /\left(p_{v} \mid a\right), \quad S_{t} \geq S_{0} ; \quad\left(p_{A} \mid a\right) /\left(p_{V} \mid a\right), \quad S_{t}<S_{0}
$$

The conditional expected time spent in transient states per visit. We obtain $\mathbb{E}\left[T_{V} \mid a\right]$ by noting that $T_{V} \mid a$ is also geometrically-distributed. Therefore $\mathbb{E}\left[T_{V} \mid a\right]=\left(p_{C} \mid a\right)^{-1}$, where $p_{C} \mid a$ is the probability that the mutant population size 
changes on a time step, conditional on fixation:

$$
p_{C}\left|a=p_{X \uparrow}\right| a+p_{X \downarrow} \mid a .
$$

We find $p_{X \uparrow} \mid a$ by Bayes' rule, and we evaluate the likelihood by inserting $S_{0}=S_{t}+1$, $a=N$, and $b=0$ into Eq. 5 .

$$
p_{X \uparrow} \mid a=\frac{\operatorname{Pr}\left(S_{T}=a \mid X_{t}=1\right) p_{X \uparrow}}{\operatorname{Pr}\left(S_{T}=a\right)}=\frac{r^{-S_{t}-1}-1}{r^{-N}-1} \frac{p_{X \uparrow}}{\alpha}=\frac{r^{-S_{t}-1}-1}{r^{-S_{t}}-1} p_{X \uparrow} .
$$

We find $p_{X \downarrow} \mid a$ analogously:

$$
p_{X \downarrow} \mid a=\frac{r^{-S_{t}+1}-1}{r^{-S_{t}}-1} p_{X \downarrow} .
$$

Inserting our expressions for $p_{X \uparrow}$ and $p_{X \downarrow}$ :

$$
\mathbb{E}\left[T_{V} \mid a\right]=\frac{1}{p_{C} \mid a}=\frac{N F_{t}}{S_{t}\left(N-S_{t}\right)} \frac{1}{(r+1)}
$$

The conditional expected number of changes in the mutant population size.

We can obtain $\mathbb{E}\left[C_{T} \mid a\right]$ from $\psi_{C_{T} \mid a}(\tau)$, i.e. the slopes of the black lines in Figs. 3 and 4 .

We can also obtain it by summing $\mathbb{E}\left[V_{S} \mid a\right]$ over all transient states:

$$
\mathbb{E}\left[C_{T} \mid a\right]=\sum_{S_{t}=1}^{N-1} \mathbb{E}\left[V_{S} \mid a\right] .
$$

We can now insert $\mathbb{E}\left[V_{S} \mid a\right], \mathbb{E}\left[T_{V} \mid a\right]$, and $\mathbb{E}\left[C_{T} \mid a\right]$ into Eq. 7 to obtain $\kappa_{a}$. The calculations to obtain $\kappa_{b}$ are analogous. We report them in the Jupyter notebook available as supporting information online.

Fig 6. Investigating the accuracy of our approximation for the conditional CFs of $T$. Theoretical approximations (thick solid lines) and simulation results (thin dashed lines) are plotted analogously to Figs. 3 and 4 . When $S_{0}$ is low (top row), our approximation for $\psi_{T \mid a}(\tau)$ is more accurate than it is for $\psi_{T \mid b}(\tau)$. When $S_{0}$ is high (bottom row), the converse is true. This observation holds whether $r=.95$ (left four plots) or $r=1.05$ (right four plots). $N=10$ for all plots. Scaling factors $\kappa_{a}$ and $\kappa_{b}$ are reported in each region of parameter space.

Fig. 6 compares our approximation for $\psi_{T \mid b}(\tau)$ and $\psi_{T \mid a}(\tau)$ (thick solid lines) with 173 Fourier transforms of simulation results (thin dashed lines). We ran 100,000 simulations 
of the Moran process for each of the displayed parameter settings and stored their absorption times conditional on fixation or extinction. Again, we plot the real and imaginary parts separately. Fig. 6 plots results from four regions of parameter space. In ${ }_{177}$ the left four plots, $r=.95$, and in the right four plots, $r=1.05$. In the top row, $S_{0}=2, \quad 178$ and in the bottom row, $S_{0}=8 . N=10$ for all plots. We see that our theoretical approximations match simulation results reasonably closely in all plots.

That match is closer in some plots than it is in others. The top row of Fig. 6 shows that, when $S_{0}=2$, our approximation for $\psi_{T \mid a}(\tau)$ matches simulation results very 181 accurately. But our approximation for $\psi_{T \mid b}(\tau)$ differs from simulation results noticeably, ${ }_{183}$ particularly further from the origin. The bottom row of Fig. 6 shows that, when $S_{0}=8, \quad{ }^{184}$ the converse is true.

\section{Sojourn CFs establish approximation accuracy}

We can show why the accuracy of our approximation for conditional absorption times depends on $S_{0}$ and where absorption occurs. Consider the conditional CFs $\psi_{T_{S} \mid a}(\tau)$ and $\psi_{T_{S} \mid b}(\tau)$ of the time $T_{S}$ that the Moran process spends in each transient state; the 'sojourn CFs' 5.16. We can find $\psi_{T_{S} \mid a}(\tau)$ and $\psi_{T_{S} \mid b}(\tau)$ by noting that $T_{S}=V_{S} T_{V}$, i.e. the total time spent in a state is the number of visits to that state, multiplied by the time spent per visit. Since the $T_{V}$ are i.i.d.:

$$
\psi_{T_{S} \mid a}(\tau)=\mathbb{E}\left[e^{\tau V_{S} T_{V}} \mid a\right]=\mathbb{E}\left[\mathbb{E}\left[\left(e^{\tau T_{V}}\right)^{V_{S}} \mid a, V_{S}\right] \mid a\right]=\mathbb{E}\left[\left(\psi_{T_{V} \mid a}(\tau)\right)^{V_{S}} \mid a\right]
$$

We can evaluate this conditional expectation. Using the tower property to split it, conditional on whether the process arrived at the transient state or not (i.e. $A=0$ or $A=1)$ :

$$
\begin{aligned}
\mathbb{E}\left[\left(\psi_{T_{V} \mid a}(\tau)\right)^{V_{S}} \mid a\right] & =\mathbb{E}\left[\left(\psi_{T_{V} \mid a}(\tau)\right)^{V_{S}} \mid a, A=0\right]\left(1-p_{A} \mid a\right) \\
& +\mathbb{E}\left[\left(\psi_{T_{V} \mid a}(\tau)\right)^{V_{S}} \mid a, A=1\right] p_{A} \mid a
\end{aligned}
$$

The first conditional expectation on the right hand side is 1 , since $V_{S}=0$ for states where the process never arrives. The second conditional expectation is a geometric sum 
that we can evaluate:

$$
\begin{aligned}
\mathbb{E}\left[\left(\psi_{T_{V} \mid a}(\tau)\right)^{V_{S}} \mid a\right] & =\left(1-p_{A} \mid a\right)+\sum_{V_{S}=1}^{\infty}\left[\psi_{T_{V} \mid a}(\tau)\left(1-p_{V} \mid a\right)\right]^{V_{S}}\left(p_{V} \mid a\right)\left(p_{A} \mid a\right) \\
& =1-p_{A} \mid a+\frac{\left(p_{V} \mid a\right)\left(p_{A} \mid a\right)}{1-\psi_{T_{V} \mid a}(\tau)\left(1-p_{V} \mid a\right)}
\end{aligned}
$$

Finally, $T_{V} \mid a$ is also geometrically distributed, and the CF of a

geometrically-distributed random variable is:

$$
\psi_{T_{V} \mid a}(\tau)=\frac{p_{C} \mid a}{1-\left(1-p_{C} \mid a\right) e^{\tau}}
$$

$\psi_{T_{S} \mid b}(\tau)$ is calculated analogously.

Fig. 7 plots $\psi_{T_{S} \mid a}(\tau)$ (first and third rows) and $\psi_{T_{S} \mid b}(\tau)$ (second and fourth rows) for all transient states of the Moran process with $N=10$ and $r=.95$. Again, we plot the real and imaginary parts separately. $S_{0}=2$ (top two rows) or 8 (bottom two rows). The location of the black arrows in Fig. 7 indicate $S_{0}$, and the direction that they point indicate whether the process fixed or went extinct. We compare our theoretical results with Fourier transforms of simulations (thin dashed traces), where we stored the amount of time that the Moran process spent in each transient state over 100,000 trials. Simulations and theory match extremely well because our sojourn CFs are exact, and we ran sufficiently many simulations of the Moran process to converge to them.

Fig 7. The sojourn CFs are relatively state-invariant when $S_{0}$ is low and the process fixes, and vice versa. Conditional CFs of $T_{S}$ (thick pink and gray lines) match perfectly with simulation results (thin dashed red and black lines). Location of arrows indicate $S_{0}=2$ (top two rows) or 8 (bottom two rows). Direction of arrows indicate fixation (first and third rows) or extinction (second and fourth rows). $N=10$ and $r=.95$ in all plots. When the conditional CFs are largely independent of $S_{t}$, we can accurately approximate $T \mid a \propto C_{T \mid a}$ and $T \mid b \propto C_{T \mid b}$.

Fig. 7 shows that when $S_{0}=2$ and the Moran process fixes (top row), $\psi_{T_{S} \mid a}(\tau)$ is almost identical for all transient states except $S_{t}=1 . T \mid a$ is largely independent of which states the process occupies before fixation. Instead, $T \mid a$ principally depends on how many state transitions occurred before fixation, i.e. $C_{t}|a . T| a$ and $C_{t} \mid a$ are then approximately proportional to each other, so our approximation is accurate. The same observation is true when $S_{0}=8$ and the Moran process goes extinct (Fig. 7 bottom row). When $S_{0}=2$ and the Moran process goes extinct (Fig. 7 second row), $\psi_{T_{S} \mid b}(\tau)$ 
differs substantially among $S_{t} . T \mid b$ then depends not only on $C_{T} \mid b$ but also on which states the process occupied, and how many times each state was visited, before extinction. Our approximation $T\left|b \propto C_{T}\right| b$ will be less accurate because $T \mid b$ is sensitive to the specific path to extinction, as well as the length of the path (i.e. the value of $\left.C_{T \mid b}\right)$. Collectively, these results suggest that we can accurately approximate $\psi_{T \mid a}(\tau)$ when $S_{0}$ is small, or $\psi_{T \mid b}(\tau)$ when $S_{0}$ is large.

Notice that some sojourn CFs in Fig. 7 appear very similar to each other. In fact, the sojourn CFs in the dashed blue boxes of Fig. 7 vary slightly between transient states, but $\psi_{T_{S} \mid b}(\tau)=\psi_{T_{S} \mid a}(\tau)$ for each state $2 \leq S_{t} \leq 8$. This identity arises because $p_{V}\left|b=p_{V}\right| a$, and $p_{C}\left|b=p_{C}\right| a$ for all transient states (see Jupyter notebook, supporting material online). The sojourn CFs $\psi_{T_{S} \mid b}(\tau)$ and $\psi_{T_{S} \mid a}(\tau)$ only differ from each other via the probabilities of arriving at a state, $p_{A} \mid a$ and $p_{A} \mid b$. So in the special case when $S_{0}=1$ and the Moran process fixes, or when $S_{0}=N-1$ and the process goes extinct, the process will arrive at every state. The only asymmetry distinguishing $\psi_{T_{S} \mid a}(\tau)$ and $\psi_{T_{S} \mid b}(\tau)$ is then eliminated, and the conditional sojourn CFs equal each other for all transient states. Therefore the conditional time distributions of fixation from $S_{0}=1$ and extinction from $S_{0}=N-1$ are equivalent, as reported in 16 .

All figures and simulations can be reproduced with a Jupyter notebook available as supporting material online.

\section{Discussion}

The Moran process was first introduced over 60 years ago, yet its exact conditional distributions of the time to absorption remain an open research topic [3, 4]. Those distributions are difficult to evaluate because the amounts of time that the Moran process spends in each transient state are interdependent. For example, say the state $S_{t}=5$ was visited $V_{5}=100$ times. Then we know that states $S_{t}=4$ and $S_{t}=6$ are less likely to have been visited once or twice than if we did not have that information. Since the number of visits to a state depend on the number of visits to neighboring states, so do the amounts of time that the process spends in them.

When faced with such a challenging problem as calculating conditional time distributions, we have a few options. First, we can either make simplifying 
approximations to the problem [4]. Second, we can settle for closed-form but intractable ${ }^{234}$ expressions for those distributions $[3]$ and numerically evaluate them. Third, we can $\quad{ }_{235}$ tweak the problem itself. Rather than calculate $T$, we can exactly and easily calculate $\quad 236$ $C_{T}$, and observe that those distributions are related to each other. The conditional, ${ }_{237}$ general, and exact distribution of $T$ might be difficult to obtain, but we can get a tractable proxy for it.

When they are applicable, martingales have several advantages compared to other popular approaches to analyzing evolutionary stochastic processes. Markov chain analysis seems to be the predominant method for studying these kinds of problems in the literature $4,27,36]$. The Markov chain approach constructs a matrix of transition probabilities and calculates e.g. absorption probabilities and times from it. While Markov chains are general and flexible tools, those calculations require us to evaluate recursions over all state space. Rarely, those recursions can be evaluated inductively $[27,31]$. We can calculate them by brute force if the size of state space is small enough $5,31,32,37$. But quite often they are written using sums of products over all state space $4,5,33,36$. If we consider more complicated evolutionary stochastic processes, e.g. an evolutionary graph with many partitions [8, 11,38, Markov chains quickly become an infeasible method of analysis. Martingales circumvent these issues because they are conservation statements, so we do not need to evaluate recursions over state space.

Other popular approaches to studying evolutionary stochastic processes include simulation $34,39,41$ and approximation $11,37,42,43]$. While both approaches are useful, they also have drawbacks. In principle, any well-defined stochastic process can be studied by simulating it enough. But simulation results cannot be generalized beyond its specific parameter settings. Computation time can prohibit us from evaluating a stochastic process over all parameter space, particularly if the dimensionality of parameter space is high. Approximations can yield tractable analytical expressions for quantities of interest, but they require us to take a special limit such as large population size [11] or weak selection $37,42,43$. Such results are ${ }_{262}$ only valid in those particular limits. Martingales can provide exact results that are valid over all parameter space without requiring simplifying assumptions.

The key to employing the martingale methodology is to find a quantity that is 
independent of the state of an evolutionary stochastic process. That quantity is then conserved throughout the process, so we can use Doob's optional stopping

theorem 24,25 to extract statistics of interest. There are a couple of ways to find such quantities. We can modify the process such that transition probabilities are state-independent, as we have done here by discarding time steps where the mutant population size remains unchanged. Alternatively, we can show that the expectation of some state-dependent quantity always equals 1 (a product martingale) or 0 (a sum martingale), regardless of the state 8,13. Finding such a quantity can be difficult, in which case we should revert to other methodologies such as Markov chain analysis, simulation, or approximation. But if we can find such a quantity, then we can quickly obtain elegant expressions for the global statistics of an evolutionary process. So other methodologies such as Markov chains, simulations, and approximations should not be our default approaches to studying such problems, but rather our fallback options.

\section{References}

1. Moran PAP. The statistical processes of evolutionary theory. Clarendon Press; 1962.

2. Moran PAP. Random processes in genetics. Math Proc Camb Philos Soc. 1958;54(1):60-71.

3. Ashcroft P, Traulsen A, Galla T. When the mean is not enough: Calculating fixation time distributions in birth-death processes. Phys Rev E. 2015;92.

4. Hathcock D, Strogatz SH. Fitness dependence of the fixation-time distribution for evolutionary dynamics on graphs. Phys Rev E. 2019;100.

5. Hindersin L, Traulsen A. Counterintuitive properties of the fixation time in network-structured populations. J R Soc Interface. 2014;11(99).

6. Askari M, Samani KA. Analytical calculation of average fixation time in evolutionary graphs. Phys Rev E. 2015;92.

7. Houchmandzadeh B, Vallade M. Exact results for fixation probability of bithermal evolutionary graphs. BioSystems. 2013;112(1):49 - 54 . 
8. Monk T. Martingales and the fixation probability of high-dimensional evolutionary graphs. J Theor Biol. 2018;451:10 - 18.

9. Tkadlec J, Pavlogiannis A, Chatterjee K, Nowak MA. Limits on amplifiers of natural selection under death-Birth updating. PLOS Comp Biol. 2020;16(1):e1007494-.

10. Allen B, Sample C, Jencks R, Withers J, Steinhagen P, Brizuela L, et al. Transient amplifiers of selection and reducers of fixation for death-Birth updating on graphs. PLOS Comp Biol. 2020;16(1):1-20.

11. Lieberman E, Hauert C, Nowak MA. Evolutionary dynamics on graphs. Nature. 2005;433:312-316.

12. Nowak M. Evolutionary Dynamics: Exploring the Equations of Life. Belknap Press of Harvard University Press; 2006.

13. Monk T, Green P, Paulin M. Martingales and fixation probabilities of evolutionary graphs. Proc R Soc A. 2014;470(2165).

14. Tan WY. On the Absorption Probabilities and Absorption Times of Finite Homogeneous Birth-Death Processes. Biometrics. 1976;32(4):745-752.

15. Glaz J. Probabilities and Moments for Absorption in Finite Homogeneous Birth-Death Processes. Biometrics. 1979;35(4):813-816.

16. Taylor C, Iwasa Y, Nowak MA. A symmetry of fixation times in evoultionary dynamics. J Theor Biol. 2006;243(2):245-251.

17. Wald A. On Cumulative Sums of Random Variables. Ann Math Stat. 1944;15(3):283-296.

18. Wald A. Sequential Analysis. 1st ed. John Wiley and Sons; 1947.

19. Wald A. Sequential Tests of Statistical Hypotheses. Ann Math Stat. 1945;16(2):117-186.

20. Tartakovsky A, Nikiforov I, Basseville M. Sequential Analysis: Hypothesis Testing and Changepoint Detection. 1st ed. Chapman \& Hall; 2014. 
21. Díaz J, Goldberg LA, Richerby D, Serna M. Absorption time of the Moran process. Random Struct Algor. 2016;49:137-159.

22. Monk T, Paulin MG, Green P. Ecological constraints on the origin of neurones. J Math Biol. 2015;71(6):1299-1324.

23. Lai TL. Martingales in sequential analysis and time series, 1945-1985. J Electron Hist Probab Stat. 2009;5(1).

24. Doob J. Stochastic Processes. New York: Wiley; 1953.

25. Ross SM. Stochastic Processes Second Edition. John Wiley and Sons; 1996.

26. Altrock PM, Traulsen A, Nowak MA. Evolutionary games on cycles with strong selection. Phys Rev E. 2017;95.

27. Broom M, Rychtar J. An analysis of the fixation probability of a mutant on special classes of non-directed graphs. Proc R Soc A. 2008;464:2609-2627.

28. Broom M, Hadjichrysanthou C, Rychtar J. Evolutionary games on graphs and the speed of the evolutionary process. Proc R Soc A. 2010;466:1327-1346.

29. Broom M, Rychtáŕ J, Stadler B. Evolutionary dynamics on small order graphs. J Interdiscip Math. 2009;12(2):129-140.

30. Voorhees B, Ryder B. Simple graph models of information spread in finite populations. Royal Soc Open Sci. 2015;2(5).

31. Voorhees B, Murray A. Fixation probabilities for simple digraphs. Proc R Soc A. $2013 ; 469(2154)$.

32. Voorhees B. Birth-death fixation probabilities for structured populations. Proc R Soc A. 2013;469(2153).

33. Voorhees B. Birth-Death Models of Information Spread in Structured Populations. vol. 14. Cham: Springer International Publishing; 2015.

34. Hindersin L, Wu B, Traulsen A, García J. Computation and Simulation of Evolutionary Game Dynamics in Finite Populations. Sci Rep. 2019;9(1):6946. 
35. M Altrock P, Traulsen A. Fixation times in evolutionary games under weak selection. New J Phys. 2009;11(1):013012.

36. Altrock PM, Gokhale CS, Traulsen A. Stochastic slowdown in evolutionary processes. Phys Rev E. 2010;82.

37. Shakarian P, Roos P, Moores G. A novel analytical method for evolutionary graph theory problems. BioSystems. 2013;111(2):136-144.

38. Díaz J, Goldberg LA, Mertzios GB, Richerby D, Serna M, Spirakis PG. On the fixation probability of superstars. Proc R Soc A. 2013;469(2156).

39. Barbosa VC, Donangelo R, Souza SR. Early appraisal of the fixation probability in directed networks. Phys Rev E. 2010;82.

40. Adlam B, Nowak MA. Universality of fixation probabilities in randomly structured populations. Sci Rep. 2014;4.

41. Alcalde Cuesta F, González Sequeiros P, Lozano Rojo Á. Suppressors of selection. PLOS ONE. 2017;12(7):1-11.

42. Tan S, Lu J, Hill DJ. Towards a theoretical framework for analysis and intervention of random drift on general networks. IEEE Trans Autom Control. 2015;60(2):576-581.

43. Allen B, Sample C, Dementieva Y, Medeiros RC, Paoletti C, Nowak MA. The Molecular Clock of Neutral Evolution Can Be Accelerated or Slowed by Asymmetric Spatial Structure. PLOS Comput Biol. 2015;11(2):1-32. 


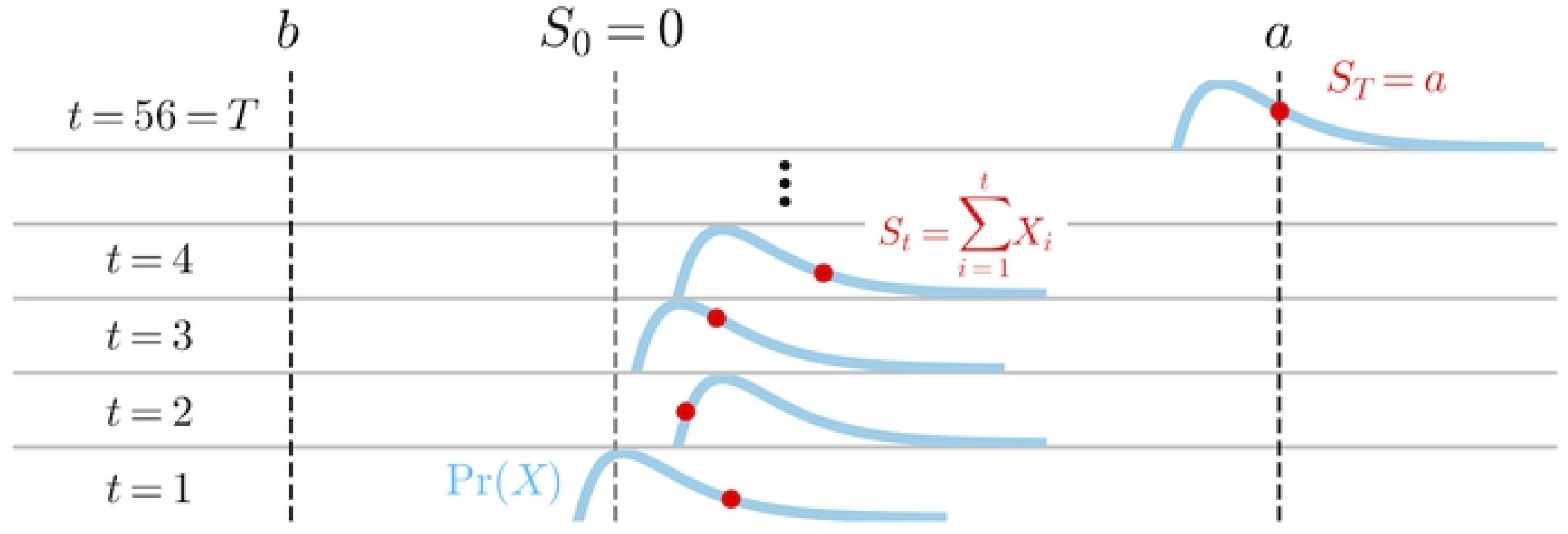

Figure 

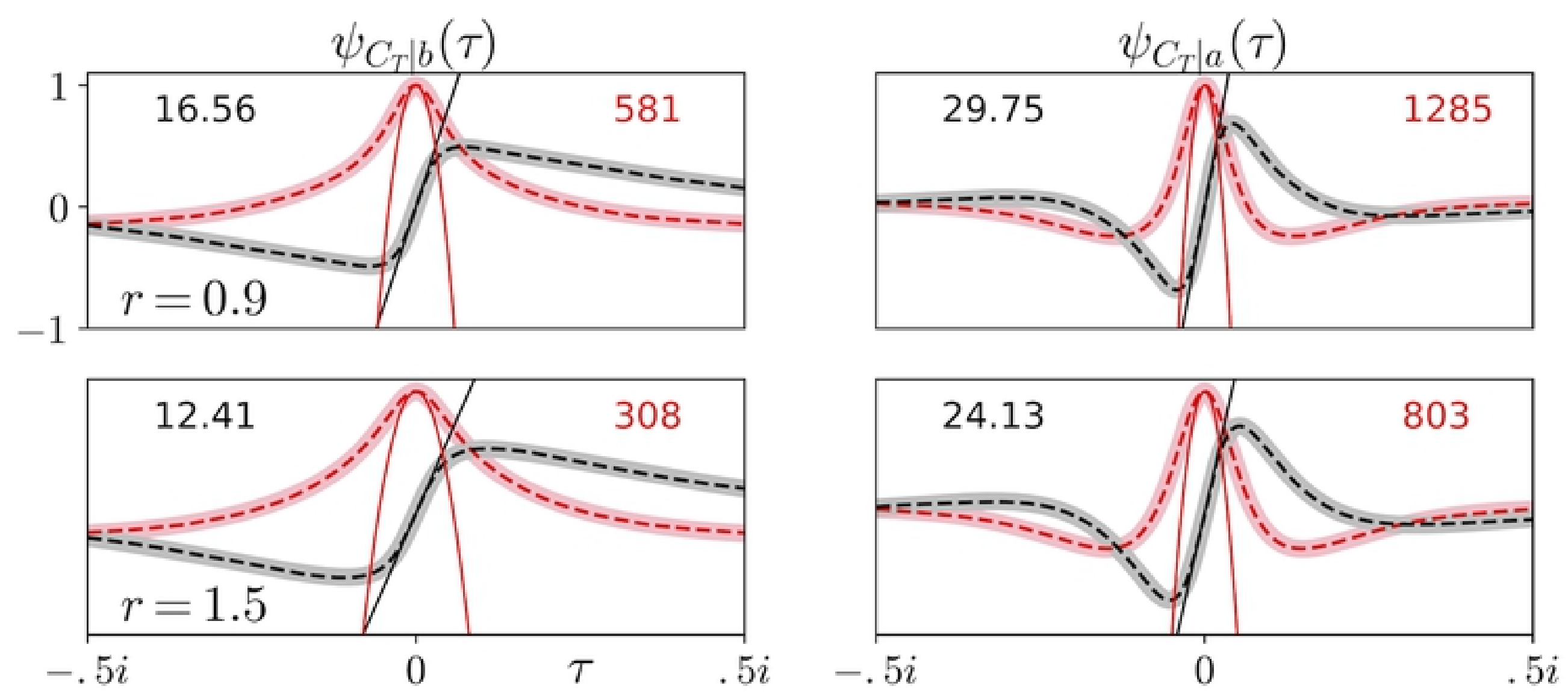

Figure 


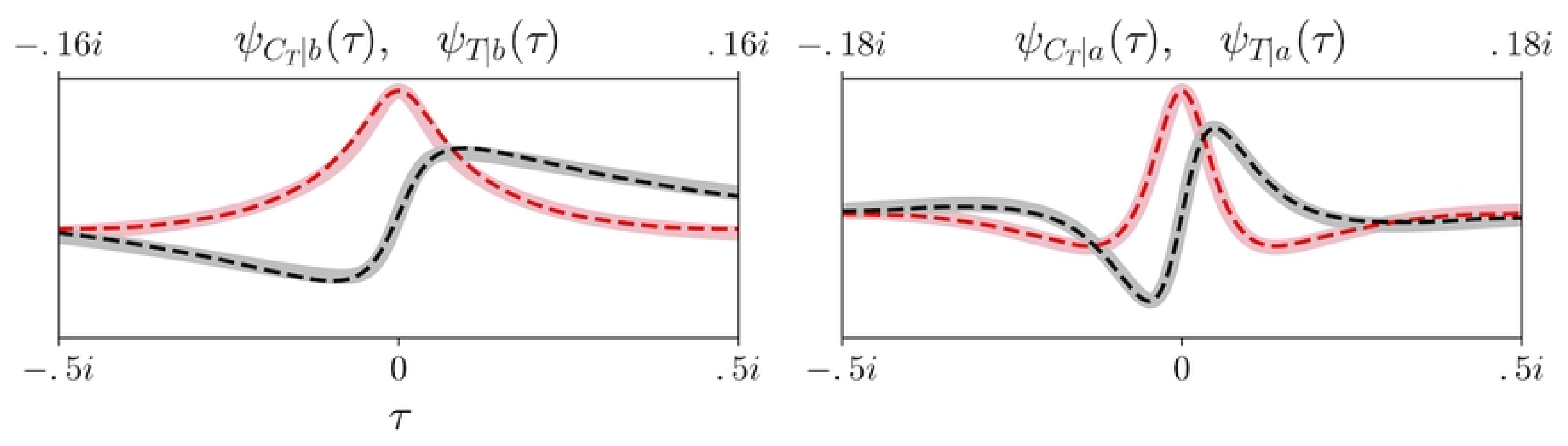

Figure 
